\title{
Capturing practitioners' "how-to" knowledge in the form of recommendations for more effective planning of collaborative adaptive management projects
}

\author{
Jim Berkley $^{1}$ and $\underline{\text { Kathi K. Beratan }}^{2}$
}

\begin{abstract}
Adaptive management (AM) and collaborative adaptive management (CAM) are concepts that are roughly three decades old, but a gap remains between theory and practice; these promising approaches largely remain more academic exercise than practical tool. Experienced practitioners report some success in applying CAM principles. Their "how-to" knowledge could be invaluable to other practitioners, but such knowledge is seldom recorded and distributed. We present results of a pilot project exploring the value and feasibility of capturing this experiential knowledge and deriving useful recommendations for more effective planning and implementation of CAM processes. We interviewed 10 practitioners from diverse backgrounds. Semi-structured interviews centered on open-ended questions that encouraged reflective narrative about experiences with a particular project. The projects described ranged widely in size and management focus. We identified 10 themes from the recommendations. The most stressed recommendation was that "getting the people part right" should be the priority consideration when setting up a CAM project. Actively engaging with communities from the very start is essential for developing practical solutions. Communities need to view the project as being consistent with community values and benefitting those communities. Supporting people with leadership qualities who are passionately supportive of the project is important for converting plans to action. Relationship- and capacity-building efforts that encourage productive interactions are essential for developing working relationships that enable implementation and long-term cooperation. Projects should be structured to take advantage of partners' particular strengths and available resources; effective and timely actions are achieved most easily at smaller scales but need to be coordinated within the context of larger issues. Great value can be obtained by simply moving away from formal implementation of AM toward actions to improve the management system's capacity to achieve success. Additional studies of smaller scale projects could provide useful information about effective approaches to capacity building.
\end{abstract}

Key Words: collaborative adaptive management; knowledge capture; practitioners; project planning

\section{INTRODUCTION}

Collaborative adaptive management (CAM) was developed to address complex natural resource management problems that are hard to resolve with standard problem-solving approaches. CAM combines adaptive management's learning and experimental aspects with collaborative management's participatory aspects (Smegstad and Gosnell 2013, Monroe et al. 2013). CAM has proven difficult to implement successfully, however (Mitroff and Featheringham 1974, Catton 1989, George 1995, Miller 1999, Susskind et al. 2010, Spradlin 2012, Chevallier 2016).

CAM concepts need to be applied by natural resource managers in real-world settings if they are to have benefits. Our objective in this work is to provide practitioners with some practical guidance in this challenging task. Currently, there is too great of a gap separating the existing body of knowledge from the resource management practitioner, so CAM remains more of an academic exercise than a practical tool. As Allen and Garmestani (2015) note, there are real shortcomings in translating theory-driven guidelines into practical, unambiguous recommendations for practitioners. A practitioner seeking information about the why and how of CAM currently comes away from the literature with two strong impressions: (1) CAM is very hard to do, and almost all previous attempts have produced disappointing results (see review in Dunning 2017); and (2) CAM is only applicable in a very narrow range of projects having a very particular and rare set of characteristics. These conclusions likely discourage practicing natural resource managers from further exploration. In contrast, we have found through interviews with CAM practitioners who have had success that it is possible to apply CAM principles and achieve positive results in real-world settings.

We began this work as a follow-up to a 2014 Special Feature in this journal put together by the Collaborative Adaptive Management Network (CAMNet; Galat and Berkley 2014). In an article summarizing the findings of the included papers, Beratan (2014) noted that the starting point for all of the papers was that it has proven extremely difficult to translate the very attractive concept of CAM into practice. She noted that the papers collectively make the case that "lack of knowledge about how to manage the human dynamics of comanagement poses a major challenge to implementing CAM." She observed that " $[\mathrm{m}]$ any of the most interesting and valuable insights gained from these projects came from the experiential knowledge of practitioners who had to work through the considerable implementational challenges" (Beratan 2014).

The practice of AM is roughly three decades old. That has been enough time for pioneering projects to reach maturity and for the outcomes of those projects to shape a second generation of CAM projects, which have reached maturity in their turn. It has also been long enough for the people involved in developing and managing those projects to reach the end of their careers and retire. Many other experienced natural resource managers have 
left or are leaving the practice now for a variety of reasons. These practitioners have valuable "how-to" experiential knowledge that should be captured before it is lost.

Sharing of this knowledge could be of great value to less experienced practitioners, but relatively little has been documented. Such how-to knowledge tends to be intuitive and context dependent, as well as difficult to articulate and thus difficult to share and to retain in organizational memory once the experienced manager is gone. This type of personal knowledge is often referred to as "tacit knowledge" (Polanyi 1958, Nonaka and Takeuchi 1995, Fazey et al. 2005, Dagenais et al. 2020). Hulme (2014) notes that many practitioners apply their own tacit knowledge in making decisions related to their work on conservation projects, particularly tacit knowledge of what is politically and administratively feasible in organizations, including local, regional, and national government bodies.

The bulk of process documentation, program evaluation, and development of best practices for CAM projects to date have focused on CAM's adaptive management components instead of the collaborative/social components. Considerable attention has been paid to development of models and understanding of the biophysical components of the natural system that can be used to estimate the likely resultant changes of specific management interventions on the biophysical components of the natural system being managed. Failure to address significant processmanagement questions has been identified as an important reason why the effectiveness of CAM efforts has been limited (Camacho et al. 2010). For example, comparatively little has been reported regarding reflective assessment of the process through which CAM projects are planned and implemented. This social process may be handled through facilitation of participant interactions, and a robust literature is available on facilitation best practices. However, most of the key interactions among project partners occur without facilitation because facilitators typically are engaged only after project planning is complete and only for particular elements of the CAM project, such as meetings with stakeholders. What is not well documented is what happens outside of facilitation: the pathways and methods of initiating a successful CAM process and the how-to knowledge of managers and decision makers who guide these processes through a project's complex multi-jurisdictional, multi-community, multi-organizational social system. Thus, we focus on the human systems aspects of CAM because of the paucity of information available in this area. Our paper's focus does not negate the necessity and foundational importance that science provides in identifying, characterizing, and guiding management in addressing problems of the biophysical system through evidence-based decision making. In this paper we present the results of a pilot project exploring the value and feasibility of capturing how-to knowledge from experienced practitioners and deriving practical and useful recommendations for the design and implementation of a CAM process.

\section{APPROACH AND METHODS}

Our research planning began with acknowledgment of the importance of relationship building in the initial stages of a CAM project. The working context for any natural resource management project is a specific social-ecological system.
Decisions and actions that influence the trajectory of that system are made by many people, organizations, and communities, most but not all of them local to the system of interest. Any effort at directed system change requires coordinated actions among many of these diverse actors. This decision/action context means that community and personal relationships are key to developing a workable solution strategy. Integrating affected people, organizations, and communities into the problem resolution process should therefore be a major focus of attention when planning projects.

The people charged with setting up and managing CAM processes typically have backgrounds in the biophysical sciences and natural resource management and have had little training in how to set up an effective collaborative process. They lack experience with the social part of the systems they manage. Resource managers typically learn through trial and error what works and what is counterproductive, as well as which actions and attitudes contribute to productive engagement by partners and stakeholders and which might shift a project in less productive directions. Over time, effective practitioners amass a considerable amount of practical how-to knowledge that they can apply in future projects.

During best practices discussions among CAMNet's Core Advisory Group, we recognized that it is difficult for practitioners to find useful guidance in the literature on how to set up an effective relationship-building process. We therefore decided to conduct a knowledge transfer project focusing on the process through which CAM projects are planned and implemented. This narrow focus allowed us to elicit information that could be compared among different project scales, focal issues, and participant roles. It is important to note that this work was not intended as a formal case study done for the purpose of research into AM-related theory. Instead, we sought a practical strategy for capturing and transferring experiential knowledge.

Our approach and methodology were drawn from diverse literatures that touch on transfer of experiential knowledge in problem-solving settings, e.g., organizational science, knowledge transfer/management, and public health and nursing. Data were collected through semi-structured interviews conducted by the authors via telephone. We used a qualitative narrative inquiry study design (Mishler 1995, Kothari et al. 2012). Our open-ended questions were designed to elicit stories about the early development of projects the interviewees had been involved with Storytelling has been found to be the most effective way to elicit implicit, contextual how-to knowledge in complex situations. Narrative stories provide explanations of how the world works (Stone 2002); they show and explain the connections between people, places, events, and things that are significant in a given context (Beratan 2007). Our approach is similar to that used by Hopkinson et al. (2017) to reflectively gain insights into the implementation of CAM from a project the interviewee was involved in.

We interviewed 10 experienced natural resource management practitioners for this pilot study; each had/has been involved in at least one project that met our criteria for classification as a CAM process (Table 1). Our initial list of interviewees included three members of the CAMNet Core Advisory Group. These 
three interviews lead to suggestions of additional interviewees who then also suggested additional interviewees. As the interviews proceeded we added to the list to arrive at the final 10 interviewees and projects.

Table 1. Criteria for classifying natural resource management projects as collaborative adaptive management (CAM) processes for this study. Projects of any size that met the criteria were eligible, and the project management teams did not have to explicitly identify their projects as CAM processes.

\footnotetext{
1 The focus is/was on addressing a natural resource management problem in which cooperation among multiple management organizations and stakeholders (governmental agencies at multiple levels, non-governmental organizations, business interests, communities, and individuals) is required. Neither the process conveners nor participants in the effort need to have identified it as a CAM process.

2 There is/was disagreement about the best management approach both because of scientific uncertainties and competing problem definitions reflecting differences in values and interests among the various management and stakeholder groups.

3 When the effort began, there was an explicit intent to evaluate the effectiveness of management activities and modify the management plan based on that evaluation.

4 The effort is either completed or mature enough to allow for assessment of the effectiveness in achieving process goals, such as fostering cooperation among key actors and moving from planning to implementation of appropriate management interventions.
}

The progression of the questions (Appendix 1) was intended to focus interviewees on their experiences with a particular CAM project and to reflect on how participants' early interactions affected the project's success. After each interviewee had spent time thinking about and describing the planning process and outcomes of a particular project, we asked what recommendations they had for practitioners just getting started with organizing an AM project. These recommendations are narrative summaries of the lessons the interviewees learned through their own experiences. We chose not to summarize or code the responses so that we could preserve the rich and nuanced information captured in the interviewees' phrasing and narrative structure. Instead, we simply transcribed the recommendations from each interview and then sorted the recommendations into topic categories or "themes."

\section{THEMES IDENTIFIED FROM INTERVIEWS}

\section{Interviewees and projects}

We recruited interviewees representing a range of training and work experience (Table 2). The projects they described ranged from small to extremely large and included a variety of ecosystems and issues being addressed. An initial set of interview subjects was suggested by members of CAMNet's Core Advisory Group. Additional subjects were identified through a "snowball sampling" approach, with each participant asked to suggest additional potential participants.

\section{Themes}

Ten themes (Table 3) emerged when we grouped recommendations into topic categories. This section includes a summary description of each theme, listed in order of decreasing importance to the interviewees, along with illustrative excerpts from the interview responses. Recommendations for each theme, transcribed verbatim from the interviews, are presented in Appendix 2; we strongly recommend reading these recommendations along with the following theme summaries.

Theme 1. Getting the "people part" right is critical.

Natural resource management is fundamentally about how people interact and make decisions. This means that the most critical part of setting up a CAM effort is getting the key people and organizations working together to identify and work toward common goals. Selection of key people, who may be the best fit for building relationships and facilitating solving the problems in a particular CAM project setting, is often constrained by factors such as managing agencies' institutional cultures, various laws, rules, and funding programs in particular. Benson and Stone (2013) noted the potential disconnect between adaptive management theory and practice relative to legal requirements and processes in the United States, which makes it difficult to successfully implement CAM. The authors acknowledge these constraints and believe our work indicates that design of approaches for the future may need to address these institutional and legal constraints for CAM. Attention to building trust is essential; facilitation can help with this. Theme 1 was by far the most commonly emphasized recommendation.

Theme 2. Projects should address meaningful problems, focus on practical solution strategies, and have well-defined goals.

Projects need to address real and acknowledged problems. "Real and acknowledged" in this context means that the problem is salient (meaningful) to both the natural resource managers and to the communities in which the project will take place. To identify and frame such a problem, the natural resource managers have to understand the communities' perceptions of the resource's value in addition to what the science of the natural system indicates so that these perspectives can be integrated where possible. This information is not only essential for gaining community buy-in and support for the project, it also can provide important information about critical social dynamics within the community, the knowledge of which can potentially benefit the ability of the natural resource managers to achieve their natural system goals. Once the problem with the natural systems has been defined, the overall goals should be generated collaboratively with robust stakeholder participation through the integration of the communities' perceptions of the resource's value and the scientific characterization of the natural system's problems to ensure that the science is directly relevant to the project's solution strategies and goals.

Theme 3. Committed involvement of people from the communities where the project will take place is important to achieving successful outcomes. This requires meaningful stakeholder participation in framing the problem and identifying potential solution strategies. Diverse viewpoints need to be included from the start.

Community engagement is critical to the ultimate success of a CAM project. Not only is it important for the community to buy into whatever solution strategy is selected, local knowledge about the community and about the interactions between the community and the natural resource is essential in identifying a plausible solution strategy. Collective identification of problems and potential solutions lends credibility and legitimacy to CAM 
Table 2. Characteristics of the interviewees and the projects they described. The ID numbers are used in Appendix 2 to identify recommendations from each interviewee.

\begin{tabular}{|c|c|c|c|}
\hline ID & Interviewee Background (Organization) & Project Scale & Project Goal / Issue \\
\hline 1 & Ecology (university) & Very large $\left(852,000 \mathrm{~km}^{2}\right.$ basin $)$ & River restoration / Invasive Species \\
\hline 2 & Environmental Policy (university) & Large $\left(88,675 \mathrm{~km}^{2}\right.$ basin $)$ & $\begin{array}{l}\text { River management for multiple uses / Native } \\
\text { species conservation }\end{array}$ \\
\hline 3 & Environmental Management (USACE) & Large $\left(28,970 \mathrm{~km}^{2}\right.$ basin $)$ & Monitor Everglades restoration \\
\hline 4 & Civil Engineering - water (USACE) & Large (166 km long stretch) & River restoration / Remove modifications \\
\hline 5 & Horticulture Agent (cooperative extension) & Intermediate (500 $\mathrm{km}^{2}$ area) & $\begin{array}{l}\text { Landscape-scale ecosystem management / } \\
\text { Landowner engagement }\end{array}$ \\
\hline 6 & Wildlife Biology (BLM) & Intermediate ( $322 \mathrm{~km}^{2}$ area) & $\begin{array}{l}\text { Development of required Land Management } \\
\text { Plan }\end{array}$ \\
\hline 7 & Natural Resource Sociology (non-profit) & Intermediate (274 km² area) & Reduce risk of catastrophic wildfires \\
\hline 8 & Ecology (USF\&W) & Small (97 km long stretch) & $\begin{array}{l}\text { Restore and protect habitat / Exotic species / } \\
\text { Fire risk }\end{array}$ \\
\hline 9 & Forest Management (State Forestry) & Small (32 km long stretch) & $\begin{array}{l}\text { Cooperative riparian zone, floodplain } \\
\text { management / Invasive species }\end{array}$ \\
\hline 10 & Urban Planning and Forestry (non-profit) & Small (60 km² basin) & $\begin{array}{l}\text { Improve water quality, stream ecosystem } \\
\text { integrity in urban stream }\end{array}$ \\
\hline
\end{tabular}

Table 3. Themes identified from the interviewees' recommendations, listed in order of importance.

\begin{tabular}{|c|c|}
\hline 1 & Getting the "people part" right is critical. \\
\hline 2 & $\begin{array}{l}\text { Projects should address meaningful problems, focus on practical } \\
\text { solution strategies, and have well-defined goals. }\end{array}$ \\
\hline 3 & $\begin{array}{l}\text { Committed involvement of people from the community (or } \\
\text { communities) where the project will take place is important for } \\
\text { achieving successful outcomes. This requires meaningful } \\
\text { stakeholder participation in framing the problem and identifying } \\
\text { potential solution strategies. Diverse viewpoints need to be } \\
\text { included from the start. }\end{array}$ \\
\hline 4 & Leadership and active engagement at all levels is needed. \\
\hline 5 & $\begin{array}{l}\text { Building and maintaining supportive relationships requires a } \\
\text { thoughtful approach to communication. }\end{array}$ \\
\hline 6 & $\begin{array}{l}\text { Scale, both of the particular problem being addressed and of the } \\
\text { organizations and communities engaged in the process, should } \\
\text { shape project design. }\end{array}$ \\
\hline 7 & $\begin{array}{l}\text { Getting to positive outcomes and achieving long-term goals } \\
\text { requires a committed champion that can keep the project on } \\
\text { track over time. }\end{array}$ \\
\hline 8 & $\begin{array}{l}\text { Consider both the long- and short-term when setting up the } \\
\text { project's structure. }\end{array}$ \\
\hline 9 & $\begin{array}{l}\text { Both organizational and cultural change take time. Small } \\
\text { successes along the way are important for keeping people } \\
\text { engaged with the process. }\end{array}$ \\
\hline 10 & $\begin{array}{l}\text { When selecting and setting up early activities, focus on capacity } \\
\text { building. This includes being flexible enough to take advantage } \\
\text { of developing opportunities and to recover from setbacks. }\end{array}$ \\
\hline
\end{tabular}

efforts and can increase chances of progress, success, and development of durable solutions. Once the problem is appropriately framed, a focus by the project on identifying practical solution strategies will foster productive community engagement. A key component of the community engagement process is building working and personal relationships and leveraging already established relationships. There are of course instances in which not all stakeholders are participating in good faith. This can result in obfuscation and obstruction to solving the problems at hand, especially when those who want to obstruct have greater power than others in the process. Our respondents did not articulate such a problem during their interviews.
Theme 4. Leadership and active engagement at all levels is needed.

Active engagement at all levels is needed to achieve success for both agencies and communities. Key decision-makers, including the project partners' leadership, need to be fully informed of what is going on and fully supportive of the project. A robust engagement process that connects the natural resource managers and the relevant local communities should be a planning priority. An initial problem assessment is needed to identify who needs to be involved, and the assessment process needs to be vetted by the involved communities. It is hard to get collaborative adaptive management started where there is existing polarization around contentious issues, and sustainable progress cannot result from trying to avoid conflict by simply picking participants who are in favor of the project. Projects thus can benefit from upfront inclusion in planning of social scientists and facilitators with conflict resolution experience.

\section{Theme 5. Building and maintaining supportive relationships requires a thoughtful approach to communication.}

Communicating in a way that is understandable to and accepted by both the natural resource management and local community cultures is important in the development of an initial understanding between these groups. Tools and methods are available that can facilitate development of shared understanding during early trust-building interactions and can contribute to a common understanding of the credibility, relevancy, and legitimacy of participants' knowledge and viewpoints, which is necessary for effective collaboration. An initial focus on common goals and interests can set a productive tone from the start, and early successes, even small ones, can validate the choice to participate and lead to expanded collaboration in the future.

Theme 6. Scale, both of the particular problem being addressed and of the organizations and communities engaged in the process, should shape project design.

Different types of actions are needed at different geographic scales and governance levels. This means that scale is an important consideration when planning a CAM project and should shape project design. Collaboration and direct action are easier to achieve in small-scale efforts with a limited number of key 
stakeholders; smaller scale efforts need to fit within the context of the larger scale program to achieve resource-wide goals.

Theme 7. Getting to positive outcomes and achieving long-term goals requires a committed champion that can keep the project on track over time.

CAM projects are more likely to succeed if they have a leader who is a strong champion of the work (Howell and Higgins 1990, Gattiker and Carter 2009). Effective project leaders have a broad perspective of the situation and understand the interconnectedness of the natural resource management challenge and the communities' concerns. They understand that leadership largely depends on inspiring others and are able to earn the trust of the diverse participants and manage with a light touch. They need to be good communicators. This type of leader can help a project overcome participants' tendency to fall back into established and unproductive patterns over time.

Theme 8. Consider both the long and short term when setting up the project's structure.

The project's structure needs to support and connect both the long-term goals and the short-term successes critical for building the capacity to do more. Short-term successes are critical to maintaining flows of resources and support from decision makers and funders, who typically need to show progress within temporal cycles that are short relative to rates of change in the natural resource. Unavoidable knowledge gaps and changing conditions mean that the project needs to be structured in a way that provides long-term stability yet is nimble and flexible enough to allow the project to take advantage of identified opportunities and to quickly recognize and address developing problems. Project leaders need to communicate the concept that adaptation can be an opportunity rather than a threat. The "adjust" step of AM contributes to the needed flexibility and should be treated as an integral part of the project plan rather than as an afterthought (Fig. 1).

Fig. 1. Diagram of the adaptive management process. Image reproduced from Adaptive management: the U.S. Department of the Interior technical guide (Williams et al. 2009:5).

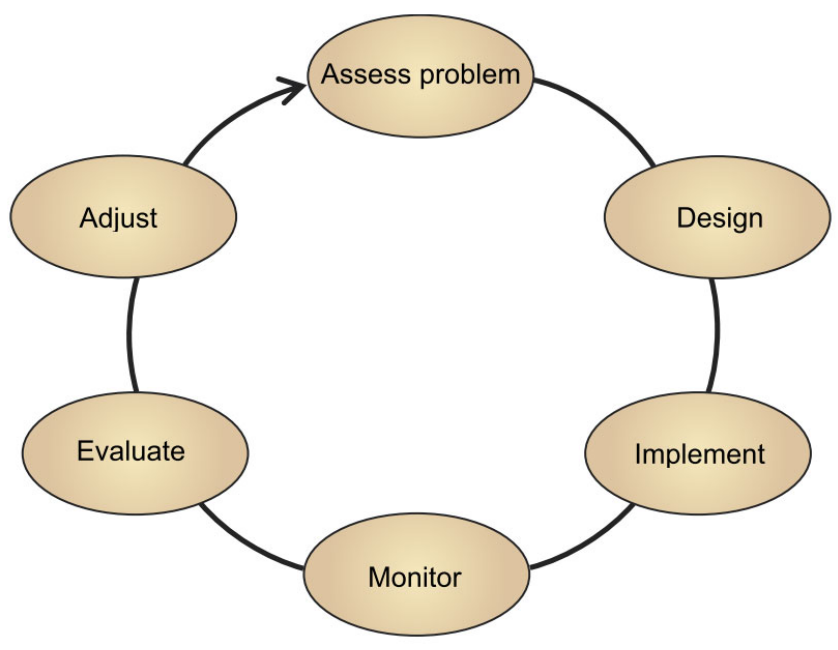

Theme 9. Both organizational and cultural change take time. Small successes along the way are important for keeping people engaged with the process.

CAM requires both organizational and cultural change in response to lessons learned: adjustments in natural resource management approaches, in the organizational cultures of natural resource management agencies, and in community expectations. It takes time for these changes to take root and get established. Projects need to grow organically and at a sustainable rate to achieve long-term success; projects should start with small, doable objectives that provide early "wins" to demonstrate to stakeholders and partners that success is possible. This type of long-sighted strategy will be most effective if the decision process is collaboratively developed by the natural resource managers and local communities so that expectations are clear and appropriate and the process is perceived as legitimate by all participants.

Theme 10. When selecting and setting up early activities, focus on capacity building. This includes being flexible enough to take advantage of developing opportunities and to recover from setbacks.

Capacity building should be a primary target for CAM projects. An increase in the capacity of communities and organizations to act together in productive ways is in and of itself a positive project outcome as well as being a leading indicator of positive changes in the natural resource being managed. Capacity has many components, including the capacity to respond nimbly when new information surfaces and adaptation is needed, to be resilient in the face of setbacks, to be able to develop problem solutions that are both creative and practical, and to have the resources needed to act and achieve goals.

\section{DISCUSSION AND SPECULATION}

The most important take-away for practitioners from our synthesis of the interviewees' recommendations is that there is real value in taking time up front to reach out to communities and work on building trust (Themes 1, 2, 3, 4, and 5). Focusing from the beginning on actively engaging with and integrating communities into efforts to resolve natural resource management problems is essential to the development of practical solutions. Our interviewees articulated that investing time and resources up front can provide large payoffs down the road; in fact, such investment is critical to actually getting anything done. We found that in our sample of 10 projects, the most effective projects emerged from up-front work in which the project team sincerely demonstrated that they care about community values and concerns, the project team found ways for the project to fit in with and build on what is going on in the community, and trust-based relationships were built. Trust and mutual respect are critically important for successful collaboration (Dale and Armitage 2011), and it takes considerable time and focused effort to build trustbased working relationships. Our interviewees suggested that the first thing a project manager should do when starting a project is to take time to listen to and learn about the local communities. Critical information includes how the communities view the resource of interest, what they value and are concerned about, which local organizations are doing related activities and might be potential partners, and which other groups need to be involved in decision making. 
Despite the importance of early engagement, the authors' experience is that the big federal and state natural resource agencies tend not to make these up-front investments of time and resources that do not initially produce concrete results. They fail to recognize that such activities make it possible for a process to get to positive outcomes more quickly (or at all), thus more than justifying the investment. The agencies include language about working with stakeholders in their documents, but their procedures indicate that this approach is not well understood and has not been integrated into the organization's culture and expectations. In practice, communities tend to get only limited attention when AM projects are planned. The narratives offered by this study's interviewees along with our own experience suggest that federal and many state-level agencies are not comfortable dealing with communities and community interests. Typically, important decisions are made by the lead organization and a small set of primary partner organizations with a pre-set list of interest groups invited to join in a stakeholder process intended to gain support ("buy-in") for those decisions and with only very limited input into the decision process.

The authors' experience with the U.S. Army Corps of Engineers (USACE) provides an example of this approach. The USACE has been put in charge of several large AM projects because of its long-established resource management responsibilities. The USACE's primary mission areas are related to civil engineering and construction of discrete structures. Based on this historical mandate, the USACE's organizational culture naturally tends to prioritize the particular assigned goal and any engineering aspects of a given project and assumes first that the USACE will have primary responsibility for all major decisions, and second that it will only be involved for a relatively short time period. These assumptions and priorities may be highly appropriate for an engineering project like building a bridge but are a poor fit for designing and implementing a collaborative AM effort. The project template USACE project teams follow includes a community engagement component, but the team members rarely have the necessary skills or organizational support to implement it. Typically, they come in with little information about the relevant communities and do not spend adequate time and effort to get additional information that could serve as a guide for project planning. The USACE team assumes "we don't have time for this" because they will only be there for a limited amount of time, so they tend not to involve stakeholders until the project is far along. As a consequence, USACE builds distrust with people in affected communities, who may engage in political end runs in an effort to be heard and resist actions by the USACE that they view as being against the community interests. This approach to project management is not unique to the USACE; many federal and state agencies exhibit similar tendencies.

Our interviewees suggested that the best way to begin project planning is to quickly set up opportunities for interaction among decision makers, scientists, and communities, with each participant given opportunities to describe what they do and talk about their issues, goals, and capabilities (Themes 4 and 5). The focus in these interactions should be on listening, with every participant demonstrating an honest interest in learning about the other participants. Such discussions can be most effective when conducted in the field; it can be easier for different groups such as farmers and conservationists to understand each other's challenges, gain respect for each other's specialized knowledge, and identify common concerns when walking through a landscape together. Transparency and effective communication are important considerations in project planning.

In addition to assisting with relationship building, the knowledge gained through such interactions can be very useful in the project's strategic planning (Themes 6, 8, and 10). One key to long-term project success is to structure a project so as to match assigned organizational responsibilities with organizational strengths and resources. The project team should apply what they learn about the priorities and capabilities of project partners, including engaged communities, to set up a project structure that takes advantage of the fact that each partner has things that they can do more effectively than any other group can. Smaller, more local organizations and local governments can contribute nuanced knowledge about the specific social-ecological systems of interest as well as local experts and volunteers who are passionate about the resource and can get things done on the ground. These groups have an intimate understanding of local conditions and opportunities and can act in ways that can alter local attitudes and behaviors to effect real and positive improvements in the resource conditions of concern. Larger organizations such as federal and state agencies and national non-governmental organizations can provide general scientific knowledge and analysis capabilities, managerial experience, and relatively stable resources. The larger organizations can keep track of the "big picture" and keep the project on track toward larger scale, longterm goals while still assisting the smaller organizations to build their capacity to act effectively over time. As pointed out by Interviewee 5, the Department of Agriculture's cooperative extension system (CES) provides a model of an effective multiscale organizational structure in which county-level offices address issues specific to local farmers, with the State office providing guidance, resources, and connections with researchers. This type of structure applied to a multi-organizational collaborative context puts the larger organizations in a more facilitative or supportive role than in a dominant actor role. This is different from the way projects have tended to be organized and may require a real change in organizational cultures.

Changing organizational culture and expectations can be very difficult and the process takes time (Theme 9), but our interviewees' experiences suggest that even small, incremental changes can produce improved results if managers are willing and able to take advantage of them. This underscores an important observation: CAM is not an "all or nothing" proposition. A particular place dealing with a specific problem may not be ready for a full CAM process. However, resource managers who think strategically and long-term can take advantage of opportunities to move things forward by making incremental progress in a productive direction (Theme 10).

Among the project manager's objectives for the information gathering and planning processes should be identifying and supporting people with leadership qualities who are passionately supportive of the resource and of the project (Theme 7). Such people are important for project sustainability because they will stick with the project for the long haul and will not let the project die when setbacks are encountered. We found this to be true for multiple projects in this study. This recommendation is consistent 
with research findings in several disciplines (including organizational development, innovation, and health care) that highlight the importance of change "champions" for innovation and transformations in technology and in practice (e.g., Schön 1963, Walters 2007). Such people provide leadership in developing a shared vision and build motivation to achieve that vision. In addition to being important in getting a project off the ground, champions are essential for keeping projects going over the long term. Among the core behaviors of champions summarized from the literature by Shaw et al. (2012) are building support for the innovation by expressing a compelling vision and boosting organizational members' skills and confidence and ensuring that the innovation is implemented in the face of organizational inertia or resistance. Different champion roles come into play at different project governance levels (Taylor et al. 2011); project champions are important for implementing project-based innovations (Tushman and Nadler 1986), whereas organizational change or executive champions provide leadership, authority, and the "big picture" vision necessary for a sustainable transformation to occur (Shaw et al. 2012).

Our interviewees indicated that one of the important tasks for champions is to gain and maintain the strong organizational support required for a CAM project to succeed over the long term, e.g., a decade or longer. The champions need to clearly explain the project's change theory, i.e., the articulation of underlying beliefs and assumptions that guide the development and implementation of a strategy to eventually manifest the CAM project, to their organizations (and their immediate supervisors) in a way that drives home the importance of "getting the people part right." This needs to be accompanied by an assessment plan acceptable to the organization that is also suitable for tracking progress in the essential up-front relationship-building work. Such progress can be very difficult to document using standard metrics; suitable indicators should derive from the project's change theory and should test the key elements of the change theory (Beratan 2019, 2020).

\section{Limitations}

The main limitation of this qualitative pilot study is the small number of interviews conducted. Many more interviews would be desirable, along with inclusion of multiple people from each project where possible. Caution is therefore advised in generalizing these findings. Ten interviews were sufficient for us to see repetition of several main points, which lends credibility to the observations; however, each interview produced useful insights so more interviews would have added richness and worthwhile recommendations. A second limitation is that all the interviewees are from the United States and discussed natural resource management projects conducted in the United States. Their insights and recommendations may be largely transferable to other contexts, but that still needs to be demonstrated.

\section{CONCLUSIONS}

The most important recommendation for practitioners from our interviewees is that "getting the people part right" should be the priority consideration when setting up an adaptive management project. The interviewees emphasized that spending significant time and resources at the very start of a project to really listen to the community and build trust-based relationships can pay big dividends in real and positive outcomes over the life of the project. Affected communities need to be active participants for natural resource management problem-solving efforts to be successful. For this to happen, those communities need to view the project as being consistent with community values and bringing benefit to those communities as informed and integrated with scientific evidenced-based information. The project management team must understand what those values are and what is important to the community in order to do effective project planning, and this requires a sincere commitment to building connections with the community and really paying attention to their concerns and interests.

One way to do this early exploration is to set up opportunities for interaction among decision makers, scientists, and communities as early as possible, with the focus of these interactions being building respect by listening to each other and getting familiar with each other's interests and specialized knowledge. Another recommended method is for the project manager and core team to look for small-scale, local groups that are actively addressing related issues within the project area. Groups focusing on natural resource management issues are obvious targets, but groups addressing other issues such as public health, community development, and social justice, as well as community members who work on or with the resource, e.g., farmers, can contribute valuable information about and connections to affected communities.

The critical importance of relationship building early in a CAM process means that the project manager should immediately recruit someone with excellent social and communication skills and who is (or could be) respected by all to help get things off to a good start. Every practitioner has their particular strengths and abilities, and not everyone will be equally effective at the "people part" of natural resource management. The focus of scientific and engineering education and training is not communities. Thus, others with those skills and training should be a part of the team, professional facilitators, on equal footing.

An ancillary point highlighted by several of our interviewees is that CAM is not an "all or nothing" proposition; great value can be obtained simply by moving away from formal definition/ implementation of AM toward actions that can improve the capacity of the management system to achieve successful outcomes. A particular situation may not be a good candidate for a full CAM process, perhaps on account of already established controversies. However, increases in trust and cooperation among project participants in problem solving can help a project be more successful even if it does not achieve full collaboration and one or more completed AM cycles. Thus, even when conditions are not in place for full-blown AM or CAM, relationship-building and capacity-building efforts that can be useful as an initial basis and foundation for key actors to develop working relationships for the time when AM or CAM is ready to launch.

Another key recommendation is to structure the project in a way that takes advantage of partners' particular strengths and the types of resources available. Different governance levels are best suited to different types of actions, all of which are important to long-term project success. Effective and timely actions can be achieved most easily at smaller scale, but these small-scale actions need to be coordinated within context of larger issues. The project team should pay explicit attention to which group or individual is best suited for each task. 
We suggest that CAM researchers would benefit from studying smaller-scale local projects, most of which do not self-identify as CAM projects. We have found that these can be an excellent and under-valued source of how-to information about successful multi-party management and problem solving. The impetus for a local project may be requirements imposed by higher level government agencies (for example, water quality targets developed by state agencies in response to national-level clean water regulations), but it is up to the local authorities to develop a strategy for meeting the target in a local context of conflicting views, competing needs, and limited public sector resources. Insufficient institutional capacity typically is more of an issue for such projects than is scientific uncertainty, and cross-sector partnerships are required for success. Small-scale, local projects can thus serve as a useful source of "lessons learned" that provide valuable insights about effective approaches to building needed social and institutional capacity for positive change.

We conclude by encouraging others to interview a wide range of natural resource management practitioners to capture and share as much valuable experiential knowledge as possible. Our 10 interviews barely scratched the surface of what can be learned about what works and doesn't work when planning and implementing challenging NRM projects.

Responses to this article can be read online at: https://www.ecologyandsociety.org/issues/responses. php/12840

\section{Author Contributions:}

I had the great joy and privilege to co-author this paper with my friend and colleague, Kathi Beratan. She was supremely wise and intelligent about the technical and human worlds and how they interrelated. Thank you Kathi. -Jim.

\section{Acknowledgments:}

We would like to thank our 10 anonymous interviewees for generously sharing their time and expertise with us. We would also like to thank CAMNet's Core Advisory Group. Our particular thanks to Jennifer Pratt Miles from the Meridian Institute, both for her long leadership of CAMNet and for her encouragement and assistance when we began this project.

\section{Data Availability:}

Relevant excerpts from our interviews are included in Appendix 2. The full interviews are not being made publicly available because they contain identifying information that could be damaging if released; the interviewees were told that the recommendations would be quoted but that the background information would be confidential.

\section{LITERATURE CITED}

Allen, C. R., and A. S. Garmestani. 2015. Adaptive management. Pages 1-10 in C. R. Allen and A. S. Garmestani, editors. Adaptive management of social-ecological systems. Springer, Dordrecht, The Netherlands. https://doi.org/10.1007/978-94-017-9682-8_1

Benson, M. H., and A. B. Stone. 2013. Practitioner perceptions of adaptive management implementation in the United States. Ecology and Society 18(3):32. https://doi.org/10.5751/ES-05613-180332

Beratan, K. K. 2007. A cognition-based view of decision processes in complex social-ecological systems. Ecology and Society 12 (1):27. https://doi.org/10.5751/ES-02103-120127

Beratan, K. K. 2014. Summary: addressing the interactional challenges of moving collaborative adaptive management from theory to practice. Ecology and Society 19(1):46. https://doi. org/10.5751/ES-06399-190146

Beratan, K. K. 2019. Improving problem definition and project planning in complex natural resource management problem situations using knowledge brokers and visual design principles. Ecology and Society 24(2):31. https://doi.org/10.5751/ES-10815-240231

Beratan, K. K. 2020. Application of intervention design concepts to project planning for collaborative adaptive management of natural resources. Ecology and Society 25(1):11. https://doi. org/10.5751/ES-11324-250111

Camacho, A. E., H. Doremus, J. S. McLachlan, and B. A. Minteer. 2010. Reassessing conservation goals in a changing climate. Issues in Science and Technology 26:21-26. [online] URL: https://issues. org/p_camachol

Catton, W. R. 1989. Choosing which danger to risk. Society 27 (1):6-8. https://doi.org/10.1007/BF02695443

Chevallier, A. 2016. Strategic thinking in complex problem solving. Oxford University Press, New York, New York, USA. https://doi.org/10.1093/acprof:oso/9780190463908.001.0001

Dagenais, C., D. Dupont, F. N. Brière, D. Mena, G. Yale-Soulière, and E. M. Sweek-Cadieux. 2020. Codifying explicit and tacit practitioner knowledge in community social pediatrics organizations: evaluation of the first step of a knowledge transfer strategy. Evaluation and Program Planning 79:1-8. https://doi. org/10.1016/j.evalprogplan.2020.101778

Dale, A., and D. Armitage. 2011. Marine mammal comanagement in Canada's Arctic: knowledge co-production for learning and adaptive capacity. Marine Policy 35(4):440-449. https://doi.org/10.1016/j.marpol.2010.10.019

Dunning, K. H. 2017. Missing the trees for the forest? Bottomup policy implementation and adaptive management in the US natural resource bureaucracy. Journal of Environmental Planning and Management 60(6):1036-1055. https://doi. org/10.1080/09640568.2016.1197105

Fazey, I., J. A. Fazey, and D. M. A. Fazey. 2005. Learning more effectively from experience. Ecology and Society 10(2):4. https:// doi.org/10.5751/ES-01384-100204

Galat, D. L., and J. Berkley. 2014. Introduction to exploring opportunities for advancing collaborative adaptive management (CAM) integrating experience and practice. Ecology and Society 19(2):40. https://doi.org/10.5751/ES-06438-190240 
Gattiker, T. F., and C. R. Carter. 2009. Understanding project champions' ability to gain intra-organizational commitment for environmental projects. Journal of Operations Management 28 (1):72-85. https://doi.org/10.1016/j.jom.2009.09.001

George, R. V. 1995. Expert support for problem formulation in urban planning. Environment and Planning B: Planning and Design 22(2):175-190. https://doi.org/10.1068/b220175

Hopkinson, P., A. Huber, D. S. Saah, and J. J. Battles. 2017. A word to the wise: advice for scientists engaged in collaborative adaptive management. Environmental Management 59:752-761. https://doi.org/10.1007/s00267-017-0825-4

Howell, J. M., and C. A. Higgins. 1990. Champions of technological innovation. Administrative Science Quarterly 35:317-341. https://doi.org/10.2307/2393393

Hulme, P. E. 2014. Editorial: practitioner's perspectives: introducing a different voice in applied ecology. Journal of Applied Ecology 48(1):1-2. https://doi.org/10.1111/j.1365-2664.2010.01938. $\underline{x}$

Kothari, A., D. Rudman, M. Dobbins, M. Rouse, S. Sibbald, and N. Edwards. 2012. The use of tacit and explicit knowledge in public health: a qualitative study. Implementation Science 7(1):20. https://doi.org/10.1186/1748-5908-7-20

Miller, A. 1999. Environmental problem solving: psychosocial barriers to adaptive change. Springer, New York, New York, USA.

Mishler, E. G. 1995. Models of narrative analysis: a typology. Journal of Narrative Life History 5(2):87-123. https://doi. org/10.1075/jnlh.5.2.01 mod

Mitroff, I. I., and T. R. Featheringham. 1974. On systemic problem solving and the error of the third kind. Behavioral Science 19(6):383-393. https://doi.org/10.1002/bs.3830190605

Monroe, M. C., R. Plate, and A. Oxarart. 2013. Intermediate collaborative adaptive management strategies build stakeholder capacity. Ecology and Society 18(2):24. https://doi.org/10.5751/ ES-05444-180224

Nonaka, I., and H. Takeuchi. 1995. The knowledge-creating company: how Japanese companies create the dynamics of innovation. Oxford University Press, New York, New York, USA.

Polanyi, M. 1958. Personal knowledge: towards a post-critical philosophy. Routledge, London, UK. https://doi.org/10.7208/ chicago/9780226232768.001.0001

Schön, D. 1963. Champions for radical new inventions. Harvard Business Review 42:77-86.

Shaw, E. K., J. Howard, D. R. West, B. F. Crabtree, D. E. Nease, Jr., B. Tutt, and P. A. Nutting. 2012. The role of the champion in primary care change efforts: from the State Networks of Colorado Ambulatory Practices and Partners (SNOCAP). Journal of the American Board of Family Medicine 25(5):676-685. https://doi. org/10.3122/jabfm.2012.05.110281

Smedstad, J. A., and H. Gosnell. 2013. Do adaptive comanagement processes lead to adaptive comanagement outcomes? A multicase study of long-term outcomes associated with the National Riparian Service Team's place-based riparian assistance. Ecology and Society 18(4):8. https://doi.org/10.5751/ ES-05793-180408

Spradlin, D. 2012. Are you solving the right problem? Asking the right questions is crucial. Harvard Business Review 90(9):84-93. [online] URL: https://hbr.org/2012/09/are-you-solving-the-rightproblem

Stone, D. 2002. Policy paradox. Revised edition. WW Norton \& Company, New York, New York, USA.

Susskind, L., A. E. Camacho, and T. Schenk. 2010. Collaborative planning and adaptive management in Glen Canyon: a cautionary tale. Colombia Journal of Environmental Law 35:1-55. [online] URL: https://scienceimpact.mit.edu/sites/default/files/documents/ Collaborative $\% 20$ Planning $\% 20$ and $\% 20$ Adaptive $\% 20$ Management $\%$ 20in $\% 20$ Glen $\% 20$ Canyon- $\% 20 \mathrm{~A} \% 20$ Cautionary $\% 20$ Tale.pdf

Taylor, A., C. Cocklin, R. Brown, and E. Wilson-Evered. 2011. An investigation of champion-driven leadership processes. Leadership Quarterly 22(2):412-433. https://doi.org/10.1016/j. leaqua.2011.02.014

Tushman, M. L., and D. Nadler. 1986. Organizing for innovation. California Management Review 28:74-92. https://doi.org/10.2307/41165203

Walters, C. 2007. Is adaptive management helping solve fisheries problems? Ambio 36(4):304-307. https://doi.org/https://doi. org/10.1579/0044-7447(2007)36[304:IAMHTS]2.0.CO;2

Williams, B. K., R. C. Szaro, and C. D. Shapiro. 2009. Adaptive management: the U.S. Department of the Interior technical guide. Adaptive Management Working Group, U.S. Department of the Interior, Washington, D.C., USA. [online] URL: https://www.doi. gov/sites/doi.gov/files/uploads/TechGuide-WebOptimized-2.pdf 
APPENDIX 1. Interview guide. including introductory statement, open-ended questions, and prompts.

Thank you for making time to talk with us!

As we said in our interview request, we're working with CAMNet to document lessons-learned by practitioners who have worked with adaptive natural resource management efforts that were complex and challenging enough to require cooperation and joint effort among many government agencies at different levels, non-governmental organizations, and key stakeholders. This type of NRM effort is what we think of as "collaborative adaptive management" or CAM.

Our specific focus is on the planning and management process, particularly interactions among the people involved in the process. The biophysical science continues to be documented elsewhere; we're trying to capture hard-won and hard-to-document experiential knowledge

We would like to hear about your experiences so that we can learn how projects get started in the 'real world' and get your insights into what worked and didn't work in projects you were involved with. We are planning to take what we learn from you and put it together with what we've learned from others in a form that can help other NRM practitioners be more effective in similar projects.

Before we get started, do you have any objection to me recording this interview? The recording will just be used to ensure accuracy. Jim and I will be the only ones who will listen to it.

We will keep what you say to us confidential. We plan to aggregate and anonymize the information from all of the interviews and present the results in the form of generalized guidelines for practice. If we wish to use a quote from this interview to illustrate a point, we will contact you and ask for permission first.

Do you have any questions?

$\rightarrow$ Please tell us just a little about your professional background. How were/are you involved in natural resource management?

$\rightarrow$ Have you been involved in any natural resource management projects that fit with our study? [Refer to the list of criteria included in the information sheet.] Is there one that stands out in your mind as particularly instructive, either as a "how-to" or a "how-not-to" example?

$\rightarrow$ We'll focus on that project now. 
-- What was the scale of the effort? For example, did it focus on a 30 sq. mile watershed, a 500 sq. ft. forest management area, a much larger ecoregion, etc?

-- Please briefly describe the issue or problem that was the focus of this effort.

-- What was your role in this effort? Were you involved in the early planning of this project?

Why was this effort initiated? What was the 'trigger' for this process?

-- How did the planning effort get started? Was a particular organization or person instrumental in getting things going? Please describe the sequence of events and the key people involved in them as you remember them.

[Prompt to keep focus specific barriers to progress, with a particular emphasis on interactions among key actors]

$\rightarrow$ How was the problem to be solved defined? Who was involved in coming up with the problem description that formed the starting point for project planning? How and by whom were those people selected and recruited?

[Prompt to keep focus on process and interactions among key actors]

$\rightarrow$ What do you consider to be the most important effects and impacts (positive or negative) of this project? What factors, actions, and/or events do you think contributed most to these important effects and impacts?

[Prompt to encourage inclusion of both bio-chemical-physical and human system impacts as well as process and interactional effects and impacts]

$\rightarrow$ What useful lessons have you learned from your experiences about planning such efforts? What advice would you give other practitioners who are starting to work with such an effort?

[Prompt to keep focus on process and interactions among key actors]

$\rightarrow$ What type of training did you have that you feel best prepared you for this type of work?

$\rightarrow$ Is there anything else you'd like to add?

$\rightarrow$ Who else involved in this project should we talk to? Who from other projects?

Thank you again for being willing to help us with this project. We'll share our findings with you once we complete our first set of interviews. 
APPENDIX 2. Theme-relevant quotes from interviews. The different interviewees are designated by numbers within brackets.

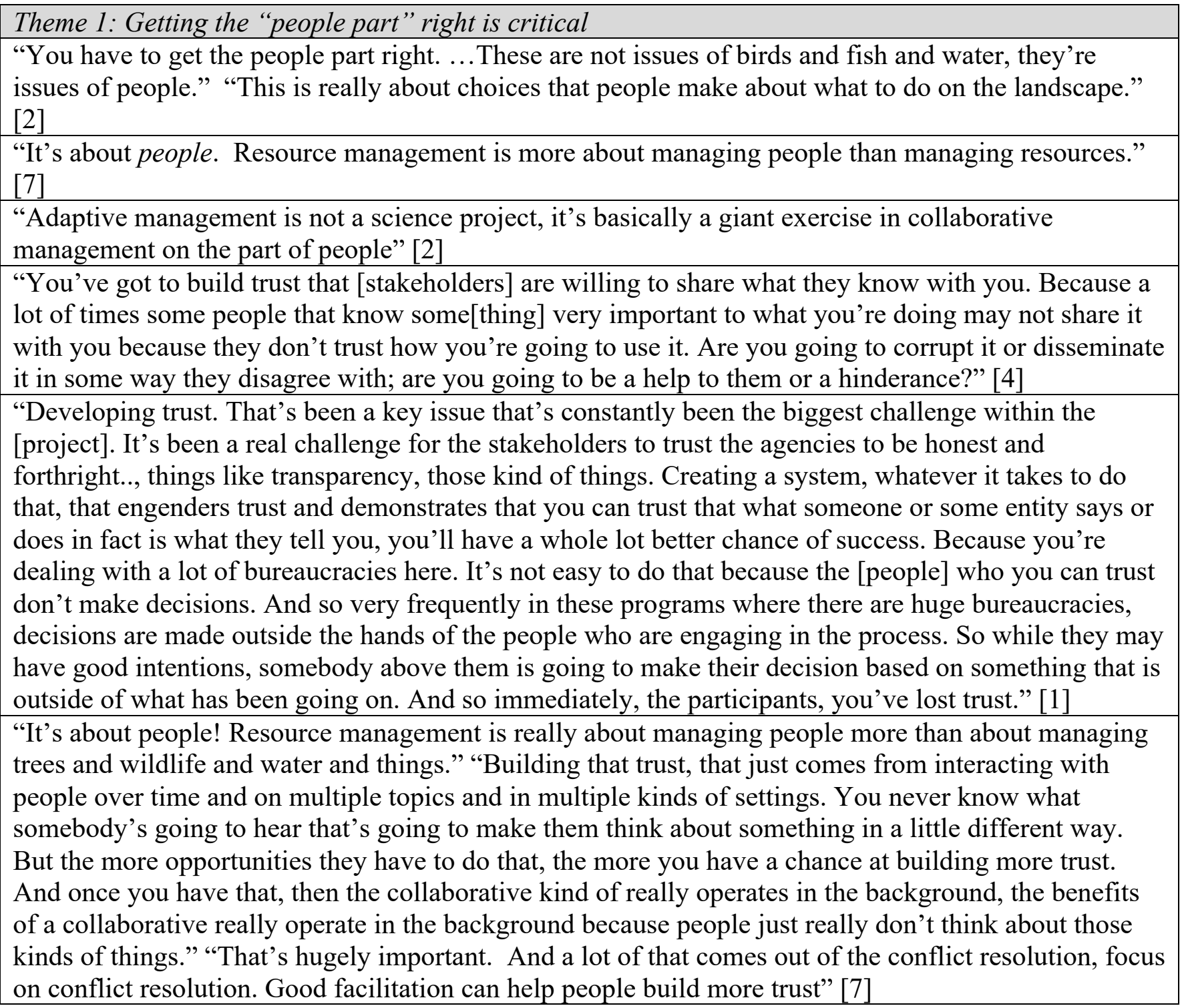

\begin{tabular}{l} 
Theme 2: Projects should address meaningful problems, focus on practical solution strategies, and \\
have well-defined goals. \\
"People are attracted to a shared vision, especially something that looks like it's solving an important \\
problem or it's part of solving an important problem." [10] \\
"Ask and answer the question of 'why?' Why are you doing this? And use that simple question to \\
help you frame what you're going to do and how you're going to do it and make sure [? what you \\
have] control over, make sure there's an awareness of the things that you don't have control over that \\
might influence what you learn or how fast you learn or your ability to learn at all. And before you \\
ever get into the science and the specifics there needs to be a goal and a set of objectives that you're \\
trying to meet and then you tailor adaptive management and your science and learning to that. This is \\
not just a big science project, anybody can do that, this is doing something to try to provide a useful \\
\hline
\end{tabular}


input to management and into decision making. So asking and answering the 'why' question is the number one thing that I've learned, that I tell people you have to do, and you have to do it well and you have to do it correctly or you will build what I call a 'science pile': that is, you'll do a bunch of monitoring and research and you'll have smart people giving really good information but [hold it in] and it sits in a corner in a pile and you can't access it and you realize that you've collected a bunch of data that have no bearing on the questions that you really need to know about. And that happens all the time." "What I've seen happen in most of these programs is the science becomes disconnected from the decision-making process. And [if you] slow things down and take the time up-front to think about what ARE our questions, what decisions do we even have control over, and what do we want to tell Governors or funders or Congress about what we're doing; got to think about that first then that'll help you figure out the technical stuff." [2]

"A lot of projects get stuck because there's not a good linkage between the technical and the policy or the political and it takes a lot of finesse to figure out how to line those things up so that all the boxes are checked so you can actually implement the project." [4]

Theme 3: Committed involvement of people from the community (or communities) where the project will take place is important to achieving successful outcomes. This requires meaningful stakeholder participation in framing the problem and identifying potential solution strategies. Diverse viewpoints need to be included from the start.

"The first thing I'd say is that - and I've seen this done formally, but I don't think it has to be done formally - of doing some kind of, you know, they've called them 'community assessments', but somehow identify some people in the area that you're going to be working on your process and your plan or your project in that can help you kind of find out who are the movers and shakers in the community, the influencers - who really needs to be involved in this process, and make sure you have this group of people engaged." [6]

Need to [start with] people who have appropriate connections [networked] to bring needed/useful partners on board. Need people on the team who aren't in silos and have lots of contacts. [5]

"A lot of the success of collaboratives is really locally based. I think you can draw on what people are doing elsewhere and bring it into a community that is building a new collaborative, but there has to be... you know, $50 \%$ of the focus has to be on that community. Just because it works someplace else doesn't mean it's going to work here. What do you guys want to do, how do you guys want to organize this, how do you guys work - if you were just going to get together with your neighbors and do something, how would you do it? What would you do? And local culture is really important. Because if you try to build something that doesn't fit in with that local culture, people aren't going to want to be involved with it and [want] nothing to do with going to another meeting and time and all that and just not want to be involved because it's not something that they really relate to and that they think is kind of enjoyable. It doesn't have to be fun, it doesn't have to be a party, but it should be something that they either enjoy or they'll take the pain because they see there's a greater benefit coming from it. If it's something that's not, it's not going to be useful. The more that these projects, processes can be things that people really want to connect to and want to be a part of, the more chance that they have for sustainability." [7]

"You CANNOT avid the issue of conflict in collaboratives; you can't avoid conflict by picking your members. Bringing together divergent views is very hard at the start, but once you work through that it becomes much better." "Even the collaboratives that seem like they're the most successful need to deal with conflict. That's where the need is for social scientists and professional facilitators who study conflict resolution, for people who can kind of come in and work through, know how to work through 
those kinds of issues. I think a lot of, I mean everybody interacts with other people all the time and I think the issue of collaboration, how to collaborate, you know when people say we're in a collaborative and we're going to do all these thinning projects, and we're going to do these prescribed burns and do these trails projects, whatever they're working on, they want to focus on those things. And they just think let's focus on those things, and we know how to collaborate because we're human beings and we always talk to each other. But there's a lot that, there's different ways you can do that, and communicate with each other and the structures you create and all those kinds of things that have to happen that those kind of influence the outcomes. Having people up front who can help you think through those issues and where you may go, and that's a lot of what I do in my job and I can tell you it's not easy because nobody wants to do that, nobody wants to sit down and go through writing a charter, or writing principles, things like that." [7]

"Engage landowners," in plans and conversations to develop the working relationships to help them recognize the problem as the manager understands it and to generate their desire to want to communicate about the common values you both have related to the resource and its problems $[8,9]$.

\section{Theme 4: Leadership and active engagement at all levels is needed}

"Having agency involvement is important, but the non-agency component is just as important. Agency folk come and go; there is a need for local leaders who are passionate about what you are doing and who will stick around. However, since local groups have no long-term funding sources, it's a challenge for them to create something sustainable, to keep things going long-term." [8]

"You have to have the buy-in from the project team level upward, all the way up into the political levels." [4]

"Having the leadership at the table all the time, involved, so that they are forced to see the sausage being made a little bit and that they are there to hear the complaints and the compliments and what's working. That's pretty important. They don't have to be there all the time, but they have to be there at the key times. They have to be engaged. And if they're not engaged and they make decisions without getting the necessary input at the level, that makes or breaks whether the program's going to work." [1] "It would be really hard to try this approach if you did not have your agency managers having your back." "I know there's people, not even within the agency, that have wanted to do some kind of a collaborative process like this around land management and they're beating their head against a wall because the agency people won't come to the table. And that always goes back to your agency manager. Because an agency manager if nothing else can make their people come to the table. But if they're afraid of turning over some of their power so that you can have a collaborative process, it's going to be very difficult for it to be successful." [6]

\section{Theme 5: Building and maintaining supportive relationships requires a thoughtful approach to} communication.

Effective communication with stakeholders is critical; how the science and engineering is presented makes a big difference in how supportive and engaged they will be. [4]

"As much as you can make it, be open to anybody that's interested and/or very transparent, I think is really important from the get-go. Lots and lots of communication and transparency about what is happening." [6]

"We've been able to not only tell people that this stuff matters but help them understand what a watershed is, where that water goes, how they and their neighborhood and in their yard or their business are connected to that. And just as importantly, give them a vision that somebody has an idea of how all this should come together. And that we can put that idea on a map and talk about it in a way 
that makes it feel like I, "I" - the citizen or the neighbor or even the person working for the local stormwater utility, can actually make a difference." "What it takes to get people in on the vision is that you actually have to go out and do something." [10]

"That was an important piece of it too from the get-go: don't focus on things that divide, focus on things that bring people together and they can more agree on at the beginning." "Focus on common goals, common interests initially. Don't come into a process and go 'oh, we've got to decide if we're going to graze or not graze this area' because you're immediately going to polarize everybody. Focus instead on "we all appreciate healthy grasslands." [6]

Theme 6: Scale - both of the particular problem being addressed and of the organizations and
communities engaged in the process - should shape project design.
"And so my suggestion to this one group was... um...to have capacity building type grants to give out
to people along your focus area or your stretch of river, in this case it was the headwaters and then
provide either one or two staff from your initiative that brings people together and that links us all and
so we can all learn from each other and so we can all grow together and I think in that way...then
if...you don't have to worry so much about the strangers coming to town telling the locals what is best
for them...um....you can more or less depend on local initiatives to make sure that you're
understanding the issues from their perspective...that being the... so the larger initiative... say on the
Missouri River effort... um...is hearing the local concerns, hearing the local issues from those local
entities and is able to kind of get a better sense of what it's like on the ground." [8]
"If you've got a big process and a big thing to conquer, take advantage of breaking into working
groups, and they can be structured any kind of different way, but those small groups are very, very
effective to actually get your tasks done." "You have to have a lot of ducks in a row to do a big project
like this and have it work, but you should not rule out, even in a fairly polarized community, that there
aren't smaller projects where you can have common goals and objectives and get a collaborative
project done." [6]
"How do you build national initiatives or regional initiatives that don't forget about what's going on
and what's successful and what's established in these small communities and support them?" "If
they're making positive change and they're moving their reach of the river along whether it's um....in
terms of health of the river or exposure to the community or community to the river...whatever they're
working on...give 'em some cash...then be that entity that brings them all together so they feel like
their shared efforts are really making a difference." [8]

Theme 7: Getting to positive outcomes and achieving long-term goals requires a committed champion that can keep the project on track over time.

It's important to have a person whose main focus of work is to drive the program. You can't expect landowners and people representing the partner organizations to do it. Landowners don't have the skill set and agencies don't have time. This person should have a LIGHT hand on the reins but should have the 10,000 foot view. This person can synergize things. [5]

Sometimes there's a benefit to having an outsider involved who isn't enmeshed in [local] entanglements, but it is essential to have a local person to serve as a community leader [in the effort. [7]

I am thrilled to know that there are people in their communities that are doing work that may not be exactly what we're doing here but they're making a positive change on the river along those different reaches of river. [8] 
First thing I'd say is, find people who are passionate. Find people who are passionate about the same thing that you're passionate about and share that passion with them and then figure out ways to link each other's missions so you can apply that passion to what you're working on. [9]

Theme 8: Consider both the long-and short-term when setting up the project's structure.

"Be comprehensive and long-term in your thinking; go ahead and try to establish a framework, but don't be afraid to take whatever the smallest part of that framework is, the biggest part that you can do and take on yourself, and just try to get wins. Don't be ashamed or afraid to do a small project because small projects turn into bigger projects." [10]

"It's really challenging to get [collaboratives] started. I think you really need to have a good structure in place early on, but everybody just wants to get to work on whatever their project is. And you know there's something to be said for having an early success., and that's going to build more buy-in to the organization and things like that, but people need to know what the organization is that they're buying into also" [7]

"Don't get too big... don't get too big too fast because you want to show accomplishment. If you want to get buy-in from other people with the same passion you got to show accomplishment. People don't want to go to meetings after meetings where you talk about the same old things." [9]

Think about the end step - the 'adjust' step - from the beginning. Think about how you will do this, including synthesis and analysis and what you're going to $d o$ with this information. People are not conditioned to do this. They want to dive right in [and do stuff], and science gets ahead of the decision process. "The science is the easy part." [2]

\section{Theme 9: Both organizational and cultural change take time. Small successes along the way are} important for keeping people engaged with the process.

"Supporting the on-the-ground-work at the community level really has to be integral to all these efforts. If you look around and I'm sure you've talked to enough entities...they're all struggling. And funding sources aren't getting larger. Foundation dollars aren't getting larger. And we're all forced to apply for these small grants that will fund us for a year or two and it's very hard to build capacity with that kind of effort. And you don't want to disappoint your community by reaching high and then failing...so grow slowly is what we've done." [8]

Community engagement is helped by, "starting small with things you can accomplish" [8]

The team "needs to get things done... if you do, people will join in", otherwise, they disengage. [9]

Theme 10: When selecting and setting up early activities, focus on capacity building. This includes being flexible enough to take advantage of developing opportunities and to recover from setbacks.

"You can make a few tiny steps forward and then there's a big setback on these projects. Now, [when there's a big setback] you can cry about that for a few minutes, but then you have to sit down and say okay, we're not going to do this project and not do the statistical rigor, we're not going to do much of the adaptive management checks and balances. What can we do in the interim to make sure this doesn't happen again and build more trust so that people use the process to solve the problem rather than play their trump card." "You always have to take what happens and figure out how you can learn and benefit from it. Otherwise, you're wasting your time." [1] Capacity building funding is needed for problem solving capacity building $[8,9]$

"All this required money. We [the board] had to find agencies that had money to do the work. So, it was pretty easy when we were just building picnic areas but now since we were changing the game and also...now that we were starting to manipulate vegetation types needed to get the Fish and Wildlife 
Service in because we were going to start to running into endangered species issues and stuff like that. All these things are things you can do. You just have to invite the right people to the table." [8] 